\title{
Poétiques migrantes dans le dernier roman de José María Arguedas : la ville à l'épreuve du métissage
}

\section{Migrant Poetics in the Last Novel by José María Arguedas: the City Undergoing the Test of Interbreeding}

Rosana Orinuela [rosana.orihuela@gmail.com]

Université de Caen, France

\section{RÉSUMÉ}

Le dernier roman de l'écrivain péruvien José María Arguedas est un texte singulier. Hors-normes, il cherche à se dérober des classifications génériques pour livrer une poétique que nous avons choisi de qualifier de «migrante ». A l'instar des flux de migrations qui redessinent et redéfinissent les rapports entre côte et sierra péruviennes, lécriture se fait, elle aussi, migrante et tente de réaliser formellement la trajectoire de migration et d'exil qu'elle décrit.

\section{MotS-CLÉS}

Peru ; Chimbote ; migration ; poétique migrante ; José María Arguedas

\begin{abstract}
The last and unachieved novel of the Peruvian author José María Arguedas called The Fox from up above and the fox from down below is a complex and plural writing. The text is plural because of the different cultures and languages that surround the Peruvian city of Chimbote. At the beginning of the 60 's Chimbote became one of the most important fishing ports of the world. Thousands of migrants from "up above" that is to say from the Andes - arrived in Chimbote converting the city in the center of a mixed migration in Peru. We propose to study how Arguedas creates a migrant poetics in order to express all the social and economical transformations that affect Peru in that decade.
\end{abstract}

\section{KeYWORDS}

Peru; Chimbote; migration; migrant poetics; José María Arguedas

REÇU 2015-08-31; ACCEPTE 2016-06-30 
José María Arguedas a ancré ses premiers écrits dans l’espace andin. Les paysages des Andes alimentent le référent géographique des romans tels que Yawar fiesta ou encore Los ríos profundos. Peu à peu, l'auteur dirige son regard vers la côte, accompagnant les mutations sociales que connaît le Pérou, ce qui lui permet d'ancrer son ouvre dans l'actualité de son temps. El Zorro de arriba y el zorro de abajo constitue le point final de cette recherche de contemporanéité : le roman tente en effet de saisir les ruptures socioéconomiques engendrées par le développement industriel des villes de la côte péruvienne. Mêlant à la fois recherches documentaires, sources ethnographiques et mythologiques et expérimentations de nouvelles formes littéraires, $E l$ Zorro ${ }^{1}$ constitue un défi pour Arguedas qui s'aventure dans l'espace de la côte, un espace qui lui est moins familier que celui de la sierra où il a vécu intimement la réalité des runas ${ }^{2}$ durant son enfance.

Modernité du texte, actualité du thème, El Zorro constitue un virage dans le cheminement artistique de l'écrivain. Dans nombre de ses lettres, la crainte de ne pas réussir à mener à bien l'entreprise de fictionnalisation de l'univers bouillonnant de Chimbote ${ }^{3}$ est à maintes reprises exprimée. Chimbote exerce, malgré tout, une fascination particulière sur José María Arguedas. Il y effectue divers voyages et reste profondément marqué par ce qu'il y découvre, pressentant que ce qui se trouve devant ses yeux constitue la pierre angulaire du devenir de la société péruvienne. Le futur s'écrit déjà dans la ville-monstre que constitue Chimbote. Dans sa correspondance, comme dans ses journaux autobiographiques, on retrouve les traces de cette fascination (Arguedas $1990: 275-295)$.

El Zorro s'écrit en lisière de la fiction et de la prétention scientifique documentaire : le romanesque chasse peu à peu la recherche de terrain ethnologique qu'Arguedas souhaitait tout d'abord mener (Arguedas 1998 : 278). John Murra rapporte en effet cette anecdote où Arguedas répond à une des critiques formulées par Aníbal Quijano - selon qui El Zorro ne reflétait pas fidèlement la réalité de Chimbote - de la façon suivante : «¡Felizmente! La novela, para ser tal, tiene que ser el reflejo de lo que soy yo. Y a través mío, si es posible, el reflejo de Chimbote " (Arguedas 1998 : 278). C'est en effectuant un détour par le mythe et la fictionnalisation qu'Arguedas tente de saisir une certaine vérité du Pérou moderne. Bien loin de sacrifier à une esthétique naturaliste, l'écrivain fusionne la précision documentaire (en témoignent les archives des interviews qu'Arguedas a menées des habitants - pêcheurs, prostituées - de Chimbote pour nourrir la construction de ses personnages) avec l'extravagance du mythe et ses recours au merveilleux.

L'imaginaire de la migration tel que le construit Arguedas dans El Zorro est traversé de dynamiques diverses : il est tantôt culturel, linguistique, poétique ou encore sociopolitique. Dans ce travail, nous nous attacherons à analyser dans quelle mesure l'imaginaire de la migration construit par l'auteur repose sur une poétique de l'hybridité.

1 Nous utiliserons désormais l'abréviation El Zorro pour désigner le roman.

2 Terme quechua utilisé par les Indiens pour se désigner eux-mêmes.

3 Ville de la côte péruvienne, Chimbote a connu un essor économique majeur dans la seconde moitié du XX ${ }^{\mathrm{e}}$ siècle. Tirant sa richesse de l'industrie de transformation de farine de poisson, entre autres, la ville s'est considérablement développée : attirant à elle des milliers de migrants, Chimbote a expérimenté les problématiques de la modernité. Arguedas y voit surtout l'occasion d'analyser la cohabitation entre les populations « criollas » (blanches), métisses, noires, et étrangères de la côte avec celles, indiennes, venues des Andes. 


\section{Histoire d'un ouvrage hors-normes}

Fruit d'une longue et ancienne réflexion sur les changements socioéconomiques qu’a expérimentés le Pérou des années cinquante et soixante, El Zorro a connu plusieurs ébauches.

Dès 1964, Arguedas travaille à l'écriture d'un grand roman qui tenterait de capter les dynamiques urbaines et économiques des villes de la côte pacifique. En partant de l'observation de la petite ville portuaire de Supe où, dans les années 1940-1950, il avait l'habitude de passer l'été, Arguedas conçoit le projet littéraire d'en dessiner les profondes mutations. Dans une lettre adressée à l'éditeur Carlos Barral et datée de 1966, Arguedas confie vouloir consacrer un grand roman aux ruptures économiques et aux bouleversements induits par l'implantation de l'industrie de farine de poissons dans la petite cité portuaire de Supe. Dans la description faite par l'écrivain, se trouvent déjà esquissées les grands lignes du futur roman pour lequel Arguedas réserve le titre de Harina mundo.

Fui testigo de la transformación del puerto y de sus gentes. De cómo esta silenciosa y paradisíaca caleta se convirtió en uan [sic] especie de urbe entremezclada de negros, cholos, indios monolingües quechuas, chyinos [sic] e injertos, prostitutas, ladrones y de empresarios sin entrañas. Varios omnibuses venían de Lima los sábados cargados de rameras y se volvían el lunes cargados de oro. El mecanismo o los métodos inventados para que esta gente informe que constituyen los pescadores permanezca siempre pobre a pesar de los increíbles ingresos que obtienen con la pesca es algo superior a las posibilidades de ficción del novelista. Pero en este horno están gentes de las costumbres más diversas: es otra imagen del Perú, en algo semejante a la que he intentado mostrar en Todas las sangres, pero más compleja aún, acaso más difícil de narrar. (Arguedas 1990 : 276-277)

Harina mundo est ainsi une première ébauche de El Zorro. Cependant, lorsqu'il découvre le formidable espace d'expérimentations de la modernité que constitue la ville de Chimbote, Arguedas modifie en substance ses projets et décide de se consacrer à la représentation de la ville-monstre. En 1967, Arguedas commence à esquisser la préparation de El Zorro (Arguedas 1990 : XXIX).

Dans son introduction à El Zorro, Eve-Marie Fell met en parallèle la représentation de la ville de Chimbote avec la résurgence mythique de la ville de Babel, allégorie millénaire de la rencontre et de la confusion des langues étrangères. La coordinatrice de l'édition évoque en effet la cité portuaire en ces termes:

Babilonia mítica de todos los lenguajes, ciudad de barriadas, de prostíbulos y de playas, de tuberculosos, locos y curas yanquis, Chimbote se nos revela al mismo tiempo en un fresco prosaico y fragmentado, a lo Manhattan Transfer, y en un baile mágico, animado por el zorro Diego, fuera de toda temporalidad racional. (Arguedas 1990 : XXI)

Cette citation a le mérite de tracer plusieurs des contours essentiels de l'œuvre : la rencontre des langues, les altérités marginales qui composent les personnages et la place du mythe dans l'espace diégétique. La mention de la «Babylone mythique » fait écho à cette mise en présence 
de plusieurs langues. Le texte stipule en effet que Chimbote constitue le centre du monde car il rassemble toutes les nationalités (Arguedas 1990 : 40). L'imaginaire de cet universalisme est activé par cette supposée présence de toutes les nationalités et, donc, de toutes les langues. Dans El Zorro, trois langues sont explicitement mentionnées comme "entendues " par les personnages : l'espagnol, le quechua et l'anglais. Les curés américains sont décrits comme parlant en anglais mais la retranscription de leurs dialogues en anglais n’est pas réalisée. Les erreurs de grammaire ou de syntaxe en espagnol de certains prêtres américains sont soulignées sans pour autant être restituées dans le texte. La présence de l'anglais est ainsi un « dit » qui n’est pas représenté : Arguedas ne choisit de «faire entendre » que l'espagnol et le quechua dans son texte, un face-à-face dont la symbolique est à analyser du côté de l'imaginaire linguistique. En parallèle de ces langues qui se frôlent, Arguedas choisit de dépeindre la ville de façon à en faire un lieu marginal et hétérotopique.

Les intentions d'Arguedas vis-à-vis de El Zorro sont claires : il s'agit pour l'écrivain de donner à voir non seulement les mutations socioéconomiques qui agitent la côte péruvienne mais également de souligner toute la valeur de la culture quechua et sa capacité à éclairer le présent le plus immédiat. Ce principe constitue par ailleurs l'assise de son engagement littéraire :

No quisiéramos que lo que hay de legendario fuera aplastado por el cemento, el asfalto y la coca cola; desearíamos que el cemento y el acero estuvieran animados y manejados con una sabiduría en que la belleza que nos viene del pasado llameara en la mente de los conductores y de los creadores, y que la sociedad se reorganizara sin matar esas mismas fuentes del pasado. (cité par Forgues $1982: 23$ )

Dans cette déclaration, se dessinent les principes sur lesquels repose El Zorro : reconnaissance de l'existence et de la richesse de la mythologie, appel lancé pour la sauvegarde des patrimoines culturels et artistiques et, surtout, mise en garde contre les dérives engendrées par cette ruée vers l'hyper-industrialisation dans laquelle est lancée Chimbote.

Avec El Zorro, Arguedas se risque à descendre des Andes, à quitter les hauts plateaux andins de ses précédents romans pour aller gratter le sable des plages polluées de Chimbote. On a beaucoup commenté et interprété la noirceur chimbotéenne comme le reflet des maux et des tourments dépressifs ${ }^{4}$ que connaissait Arguedas au moment de la rédaction de El Zorro. Ces deux réalités, la situation personnelle de l'auteur et la noirceur du roman sont certes liées, indiscutablement. Le seul fait d'intégrer les diarios au récit fictionnel permet en effet d'affirmer ce lien cependant, il n'est pas possible de déterminer le poids exact de chacun de ces éléments. Quelle est la folie première ? Celle de l'auteur ou bien celle de la ville, de la souffrance socioéconomique ? Quel mal contamine en premier l'autre ? Indémêlables douleurs, inextricables démences : Chimbote, la ville éperdue, rencontre Arguedas, l'homme tourmenté. La lutte représentée dans les diarios semble bien être celle de deux souffrances au monde : l'acculturation que vit la population indienne de Chimbote et la crise personnelle de l'auteur.

4 José María Arguedas connaît une période de profonde dépression lorsqu’il entame la rédaction de El Zorro. Une première tentative de suicide en 1966 atteste de l'extrême fragilité dans laquelle l'homme se trouvait quelques années plus tôt. 


\section{Une poétique hétérotopique}

Ville réelle en même temps qu'elle se soumet aux fantasmes de ses habitants devenant, par là-même, irréelle, Chimbote est bien plus qu'un cadre géographique référentiel et se meut en personnage romanesque. La représentation de la ville subit en effet un régime de distorsions temporelles, linguistiques et fantasmées. Dans la nouvelle ville-monde que constitue Chimbote dans les années cinquante, le national se superpose à l'international, et les langues et les cultures cohabitent.

[...] la explosión demográfica de Chimbote sólo se explica por una masiva migración cuyos protagonistas tenían las más variadas procedencias geográficas y condiciones sociales de infinita diversidad : extranjeros de múltiples orígenes, criollos costeños y afroperuanos, pero - sobre todo - indios y mestizos andinos todos mezclados en un torrente multiétnico y plurisocial. (Cornejo Polar 1997 : 269-270)

Dans le roman, la ville portuaire est saturée de seuils symboliques et réels : on distingue ainsi des frontières linguistiques - dans la ville se frôlent le quechua, l'espagnol et l'anglais - des limites géographiques - les Andes, la côte ainsi que la dualité précolombienne entre l'urin et l'hanan, une dualité par ailleurs remise en cause par les flux migratoires andins - et des frontières temporelles transgressées par la fusion entre le temps mythologique et le présent. A ces frontières " concrètes ", s'ajoutent ce que nous pourrions désigner par l'expression « les frontières de l'autre " : la peur de celui qui n'est pas semblable à soi, le racisme et la xénophobie qui gangrènent la ville. Le racisme est si présent que les individualités ne se définissent que contre une altérité rejetée : l'identité se construit ainsi par la négative. Les migrants venus de la sierra rêvent d'effacer leur origine andine pour mieux revêtir celle des criollos. Les injures raciales émaillent les discours à tel point que, derrière cette surenchère, se devine une véritable haine de soi. Ce que démontrent les tensions racistes dévoilées dans le roman, c'est le principe défini par Edouard Glissant selon lequel « toute identité sétend dans un rapport à l'Autre » (Glissant 1990 : 23). L'Un ne se définit qu'en fonction de l'Autre : l'Autre est un miroir - miroir inventé, miroir familier, rassurant ou trouble - dans lequel l'Un projette ses phantasmes. Loin d'être une donnée essentielle ou immuable, l'identité résulterait donc d'une confrontation et des rapport négociés avec l'altérité. Les altérités sont douloureuses : l'autre est rejeté pour ce qu'il représente socialement. Ainsi, les migrants serranos sont marginalisés du seul fait de leur origine : « ¡Fuera de ahí, cholo serrano desgraciado, chavetero, contramadre! ¡Saca el cuchillo, indio gallina, patrón de la put e’tu madre! » (Arguedas $1990: 40$ )

La représentation de la ville se dérobe peu à peu à une interprétation fantasmée : dans le regard de celles qui haïssent profondément Chimbote, leur description ressemble à celle d'un enfer.

Llegó a la carretera «marginal » de gruesa arena y basura en que empiezan las calles, todas derechas y en cuadro, de la barriada. Abajo, al pie del médano, el puerto pesquero más grande del mundo ardía como una parrilla. Humo denso, algo llameante, flameaba desde las chimeneas de las fábricas y otro, más alto y con luz rosada, desde la fundición de acero. No alcanzaba al cerro la pestilencia del mar. (Arguedas $1990: 44$ ) 
La mer est par ailleurs dévastée par l'exploitation intensive de la pêche et les activités industrielles d'acier et de transformation de farines de poisson : le paysage et les écosystèmes sont dégradés et leur représentation est apocalyptique. La mer se vide de ses poissons et les pélicans n’ont plus de quoi se nourrir. Affamés et moribonds, ils errent dans les marchés de la ville où les enfants leur jettent des pierres. L’odeur de la mer en décomposition est insoutenable et la plage semble, elle aussi, pourrir :

Asto salió de la huella; sus zapatos nuevos se hundieron en la arena. La fetidez del mar desplazaba el olor denso del humo de las calderas en que millones de anchovetas se desarticulaban, se fundían, exhalaban ese olor como alimenticio, mientras hervían y sudaban aceite. El olor de los desperdicios, de la sangre, de las pequeñas entrañas pisoteadas en las bolicheras y lanzadas sobre el mar a manguerazos, y el olor del agua que borbotaba de las fábricas a la playa hacía brotar de la arena gusanos gelatinosos; esa fetidez avanzaba a ras del suelo y elevándose. (Arguedas 1990 : 40)

La ville est, par ailleurs, aux mains d'une mafia qui créé un cercle vicieux, entraînant les pêcheurs à dépenser l'argent reçu de la pêche dans les bars et les bordels de la ville tenus par la même mafia que celle de la pêche. Ce cercle infernal est décrit par le personnage de l'industriel don Angel en ces termes : "Pero la "mafia" hizo gastar a los pescadores en su debido tiempo; cebó sus apetitos de machos brutos. Con buenos los hizo derrochar de todo lo que ganaban; los mantuvo en conserva de delincuencia, y esa mancha no se lava fácil » (Arguedas 1990 : 96) Dans cet asservissement programmé de la main d'œuvre migrante, se lit un profond mépris pour les Indiens que l'on peut aisément percevoir dans la citation ci-dessous :

Ningún indio tiene patria, ¿no? Me consta. No saben pronunciar ni el nombre de su provincia. Ningún cholo, ningún negro verdadero, zambo o injerto, tienen concierto entre ellos. Son peores que los indios en eso. ¿Dónde está la patria, amigo? Ni en el corazón ni en la saliva. «¡A la mierda!», es el juramento de los cholos, injertos y negros; y los indios son una manada. (Arguedas $1990: 116)$

Par ce traitement dégradant et cet asservissement dans lequel sont tenus les migrants serranos, les individualités s'estompent pour laisser place à une masse indifférenciée d'hommes réduits à nêtre plus que des bras. Leur visage s'efface ; ainsi, lorsque le syndicaliste Zavala observe la file des pêcheurs tous retenus et fascinés par l'exhibition des prostituées, il ne peut s'empêcher de penser : "No tienen cara, creo, éstos, o yo tengo los ojos hechos brea...» (Arguedas 1990 : 43). La ville-monstre parvient ainsi à ôter toute singularité pour transformer en une homogène et indistincte foule la multiplicité qui la compose.

\section{Des langues migrantes}

El Zorro dévoile une poétique de la relation : Arguedas dresse en effet les contours d'une modernité qui ne peut pas ne pas intégrer les diversités qui la composent, sous peine de sombrer. Dans le texte, les langues de la ville entrent en coïncidence. L'espace linguistique de l'espagnol 
est ainsi habité par le quechua : en témoigne, par exemple, l'espagnol teinté de quechua parlé par les migrants serranos comme Asto ou don Esteban dont le discours ne cesse d'être traversé par les variations de la langue indigène :

Me mamacita, entonces, baila bailando, si'ha ido, lejos, a incontrar el carretera qu'iba costa. Si ha llevado a so hijo chiquito; a me ya también, que era maqtillo, de ocho, diez años sería, mi’ha empojado al camino del chacra de me tío Anibal. [...] ¡Ay, compadrito, compadre! Hemos trabajado en plantación coca. "Eras niño toavía, entonces?" ha pregontado me compadre. No, hey contestado. Tendreya unos trece, catorce doce; me premo era mayorcito, maltón ya. Oiga ostí, compadrito, nus amarraban con cadena en de noche, el hacendao. (Arguedas $1990: 147$ )

Sous la langue espagnole, se dessine, sinueuse, la langue quechua. Lespagnol devient alors une langue incertaine, brouillée, et, aux yeux de certains critiques, malmenée (Forgues 1982 : 78). Arguedas réussit ainsi à " déterritorialiser » l'espagnol, pour reprendre une expression de Gilles Deleuze, c'est-à-dire à lui faire subir l'expérience de la migration. La langue des migrants serranos devient une langue hybride où l'espagnol est " hanté5 " par le quechua. Cette hybridation fait écho à la confrontation identitaire à laquelle font face les migrants andins. Leur espagnol est ponctué d'agrammaticalités, de distorsions phonétiques et d'erreurs syntaxiques à l'image de la perte de repères culturels à laquelle ils font face. Le travail que réalise Arguedas sur la langue peut s'analyser à la lumière de ce quécrit Gilles Deleuze à propos du dramaturge Carmelo Bene ; le philosophe explique, dans «Un manifeste de moins », que l'écrivain italien fait subir un traitement particulier à la langue, qu'il la minorise, c'est-à-dire qu'il lui fait faire l'expérience du mineur en lui ôtant les assises rassurantes d'une syntaxe établie et d'une grammaire acquise (Deleuze 1979 : 87-131). Dans la bouche des migrants andins, l'espagnol est, lui aussi, amputé de sa cohérence syntaxique et grammaticale. De la même manière que le faisait Carmelo Bene avec l'italien, il s'agit pour Arguedas de « faire passer toute chose par la variation continue, comme sur une ligne de fuite créatrice, qui constitue une langue mineure dans le langage, un personnage mineur sur la scène, un groupe de transformation mineur à travers les formes et sujets dominant » (Deleuze 1979 : 119).

\section{Poétique(s) migrante(s)}

Parallèlement à ces flux migratoires et à ces frontières sociogéographiques qui se trouvent balayées, se redéfinissent les moyens formels et les codes littéraires pour les décrire. Arguedas a renouvelé l'écriture romanesque péruvienne en allant plus loin que ce que le mouvement artistique et politique nommé indigénisme avait institué. L'indigénisme cherchait à dépeindre les mondes culturels indiens d'Amérique Latine en faisant pénétrer l'univers andin dans la littérature $^{6}$. Parce qu'il a partagé dans son enfance la vie des runas comuneros qui travaillaient pour

5 Pour reprendre une expression d'Alexis Nouss.

6 Voir à ce sujet l'essai consacré à l'indigénisme en littérature «La narrativa indigenista peruana » de Tomas G. Escajadillo, Lima, Amaru Editores, 1994. 
sa belle-mère, Arguedas a renouvelé les formes de l'indigénisme et la représentation du monde andin runa. Il a par ailleurs construit la totalité de son œuvre autour de cet impératif esthétique d'une représentation authentique du monde andin. Dans El Zorro, pourtant, le style change : la complexe modernité de la ville exige une écriture elle aussi complexe et expérimentale. Bien que le roman demeure inachevé, il est significatif de se pencher sur les ruptures, les ellipses et le caractère presque saccadé de la narration qui participent d'une poétique transgressive qui bouscule l'écriture arguédienne des romans précédents. Les histoires prolifèrent autour d'une multiplicité de personnages et de dialogues: Arguedas créé des espaces romanesques périphériques et refuse d'ancrer El Zorro autour d'un seul axe directeur, une façon de reproduire formellement le nomadisme de la migration (Glissant 1990 : 41). Il y a ainsi dans le texte un va-et-vient narratif, propre à retranscrire la condition migrante des personnages décrits : vaet-vient entre la fiction et le réel, le romanesque et l'autobiographique, le romanesque et le mythologique, les voyages d'Arguedas de Santiago à Lima pendant la rédaction d'El Zorro. L'hésitation et la difficulté à écrire de l'auteur, la peur de la feuille blanche, les doutes et les angoisses exprimées font que la narration peine à s'inscrire dans la continuité. De la même façon, la glose que réalise Arguedas sur la rédaction de El Zorro et la peine qu'il éprouve à mener l'écriture du récit, actualisent de façon métalittéraire le déracinement éprouvé par les migrants.

C'est par ailleurs grâce à son intertextualité que El Zorro se constitue comme pluriel, comme hybride (Cornejo Polar 1984 : 35). Lécriture migre en effet sous présente emprunts et références culturelles, de la même façon que l'espagnol migre selon les occurrences de la langue quechua. Arguedas lie le passé historique et mythologique du Pérou avec la représentation de sa contemporanéité la plus immédiate : il éclaire un monde (la modernité qui bouleverse la côte) par un autre (les figures mythologiques quechuas). Chimbote vient également buter contre le temps du mythe: elle l'interroge pour mieux en faire ressortir les couleurs du silence et de l'absence. Arguedas nourrit la représentation du port de Chimbote de références explicites aux figures mythologiques de Dioses y hombres de Huarochirí. Le texte se partage ainsi entre un temps contemporain et un temps mythologique lointain. Lieu interstitiel, Chimbote est le lieu de fusion des imaginaires mythiques et contemporains : on pensera, par exemple, à la réactualisation de la figure et la destinée de Tutaykire. Evoqué dès les premières pages du récit fictionnel, le héros Tutaykire connaît une fortune similaire à celle des hommes de la ville. Retenu par une «zorra douce et contraire » dans l'espace d'en bas (Arguedas 1990 : 29), Tutaykire ne regagne plus les Andes. De la même façon, les migrants serranos sont captivés par l'attrait et l'appel de la richesse que représente pour eux la baie de la ville appelée maintes fois par plusieurs personnages « la plus grande chatte du monde " (Arguedas 1990 : 41). En dressant de nombreuses correspondances entre les héros des récits mythiques de Huarochirí et les personnages qui alimentent la fresque de Chimbote, Arguedas réussit à faire basculer la représentation du monde de la côte à travers le prisme de la culture quechua. Cette intertextualité culturelle donne lieu à une écriture plurielle. C'est ainsi un bouleversement du regard qui s'opère : la culture quechua est le prisme par lequel s'écrit la fresque des migrants serranos venus s'installer sur la côte.

7 Mythes précolombiens recueillis en quechua par le missionnaire Francisco de Ávila au XVII siècle dont Arguedas a livré une traduction en 1966 en collaboration avec l'historien Pierre Duviols, une traduction publiée sous le titre de Dioses y hombres de Huarochirí. 
En conclusion, nous pouvons affirmer que la poétique de El Zorro est bel et bien une poétique migrante au sens où elle tente d'actualiser formellement l'expérience de migration que le texte dépeint. A la transgression formelle que constitue l'entrelacement entre fiction et autobiographie, emprunts mythologiques et fiction « post-moderne », répondent les phénomènes de migration décrits par l'auteur. Ce dernier ne souscrit pas à une esthétique réaliste mais choisit de réaliser un détour par le mythe. Ce n'est donc pas une représentation mais une interprétation du Pérou moderne que livre Arguedas :

Y no es que pretenda describir precisamente Chimbote. No ustedes, lo saben mejor que yo. Esa es la ciudad que menos entiendo y más me entusiasma. ¡Si ustedes la vieran! ¡Tengo miedo, no puedo comenzar este maldito capítulo III, de veras! (Arguedas $1990: 84$ )

Arguedas refuse ainsi le linéaire, le fixe, et accueille l'hybride. Par l'entrecroisement de cultures et de langues, l'écrivain donne à voir dans toute sa complexité le devenir de la société péruvienne. L’ensemble de son œuvre, entièrement tournée vers l'altérité, trouve dans ce dernier roman une de ses plus belles résonnances. Comme le soulignait fort à propos Corinne Mencé-Caster dans son article consacré au roman Los ríos profundos, Arguedas « invente pour la langue et la culture quechua une patrie dans la langue espagnole» (Mencé-Caster 2004 : 90).

\section{Références bibliographiques}

Arguedas J.-M. (1990). El Zorro de arriba y el zorro de abajo. Nanterre : AALCA XX. . (1998). Las cartas de Arguedas. Lima : Pontificia Universidad Católica del Perú, Fondo Editorial. Cornejo Polar A. (1984), Vigencia y universalidad de José María Arguedas. Lima : Editorial Horizonte.

- (1997). Los Universos narrativos de José María Arguedas. Lima : Editorial Horizonte.

Deleuze G. (1979). Un Manifeste de moins. In Superpositions. Paris : Les Editions de Minuit.

Deleuze G., \& Parnet C. (1996). Dialogues. Paris : Flammarion.

Escajadillo, T. (1994). La narrativa indigenista peruana. Lima : Amaru Editores.

Forgues R. (1982). José María Arguedas : de la pensée dialectique à la pensée tragique : histoire d’une utopie. Toulouse : Presses universitaires du Mirail.

Glissant E. (1990). Poétique de la relation. Paris : Gallimard.

Mencé-Caster C. (2004). La recréation de la langue véhiculaire comme réponse à la domination ressentie : les stratégies scripturales de José María Arguedas dans Los ríos profundos. In Moreno, F. (Coord.), Los ríos profundos : José María Arguedas. Paris : Ellipses. 
